\title{
Erratum to: Measurement and analysis of the shape recovery process of each erythrocyte for estimation of its deformability using the microchannel technique: the influence of the softness of the cell membrane and viscosity of the hemoglobin solution inside the cell
}

\author{
Tsutomu Tajikawa $\cdot$ Yuya Imamura $\cdot$ \\ Takaya Ohno $\cdot$ Fumiya Muranishi . \\ Maki Kubota $\cdot$ Kenkichi Ohba
}

Published online: 31 August 2012

(C) Japanese Society of Biorheology 2012

Erratum to: J Biorheol

DOI 10.1007/s12573-012-0052-9

The article cited above was incorrectly categorized. The correct category is Original Article, not Review Article.

The online version of the original article can be found under doi:10.1007/s12573-012-0052-9.

T. Tajikawa $(\bowtie)$

Department of Mechanical Engineering,

Faculty of Engineering Science, Kansai University,

3-3-35, Yamate-cho, Suita, Osaka 564-8680, Japan

e-mail: tajikawa@kansai-u.ac.jp

Y. Imamura - T. Ohno · F. Muranishi · M. Kubota

Graduate school of Science and Engineering,

Kansai University, Suita, Japan

K. Ohba

Organization for Research and Development of Innovative

Science and Technology, Kansai University, Suita, Japan 